\title{
Design and realization of a broadband single-side-band mixer with a very short settling time
}

\author{
S. Haßler ${ }^{1, *}$, T. Reichthalhammer ${ }^{1}$, and E. Biebl ${ }^{1}$ \\ ${ }^{1}$ Fachgebiet Höchstfrequenztechnik, Technische Universität München, Munich, Germany \\ *now at: Siemens AG, Erlangen, Germany
}

\begin{abstract}
To achieve high range resolution in synthetic aperture radar imaging a frequency synthesizer with high bandwidth is a possible solution. To operate in the required frequency band an LF-signal usually has to be upconverted. In this paper we describe the design and realization of a broadband Single-Side-Band Mixer with a very short settling time between frequency steps of arbitrary length inside a high bandwidth. Compared to already existing SSB-Mixers, our novel concept has three major advantages: At first, the mixer could be used in combination with an arbitrary signal source. Due to a modular circuit concept it is possible to use the system for different input frequency ranges. Moreover, just by changing single modules, the output frequency-range can be adapted to individual requirements. Thirdly, as a main advantage, the system is able to generate a high frequency output span with a very fast settling time between frequency steps. Even with applied steps up to $400 \mathrm{MHz}$, the settling time remains below $3 \mu \mathrm{s}$, which is more than 5 times faster than the settling time of similar synthesizers.
\end{abstract}

\section{Introduction}

A few requirements have been claimed for developing the single side band mixer: A fast frequency sweep, a high output spectrum and a high sideband supression. For synthetic radar imaging with a stepped frequency synthesizer, for example, frequency ramps have to be generated and therefore the system has to be able to switch very fast between discrete frequency steps of up to $400 \mathrm{MHz}$. Within this time the sideband suppression has to rise as much as possible. Due to a modular concept, the system could be adapted to different signal sources and various RF-frequency-ranges (Haßler, 2009).

Correspondence to:

T. Reichthalhammer

(reichthalhammer@tum.de)

\section{System design}

There are different concepts how to convert frequency with a high sideband suppression. In Fig. 1 there is shown a fundamental circuit concept of our mixer. The signal is devided into two channels with a relative phase difference of $90^{\circ}$.

The signals amplitude is equalized by an AGC. Due to the requirement of a very fast settling time, the time-constant of the AGC is a fundamental criterion of choice. A broadband AGC unit is necessary to allow a high input spectrum. The output level of the AGC is compared to the setpoint and the difference converted into a current which charges an external capacitor. The voltage at the capacitance adjusts the amplification factor. The smaller the capacitance the lower the settling time but also the higher the probabilty that the system oscillates. With our system we achieve a settling time of $1 \mu \mathrm{s}$. The influence of the amplitude differences between the two signal paths on the sideband suppression has been evaluated using a simulation. To show the influence of amplitude differences between the two signal paths on the sideband suppression, the single sideband mixer was simulated. For this simulation, a phase difference of $90^{\circ}$ between the I- and Qsignal was assumed. Figure 2 shows the output spectrum of the single sideband mixer during the settling time after a $400 \mathrm{MHz}$ step. Figure 3 shows the output spectrum after the settling time. The different sideband suppression is obvious.

Each signal is mixed with the VCO signal, that is splitted in an inphase and a quadrature component as well. Assuming an equivalent signal level and a phase difference is $90^{\circ}$ Fig. 4 shows the spectra of the input signals AI1 and AQ1 together with the output signal.

\section{Amplitude and phase error}

Referring to Fig. 1, the input signals AI1 and AQ1 can be written as

$A_{I 1}(t)=A_{I 1} \cos \left(w_{\text {in }} t+\Delta \Phi_{I}\right)$

Published by Copernicus Publications on behalf of the URSI Landesausschuss in der Bundesrepublik Deutschland e.V. 


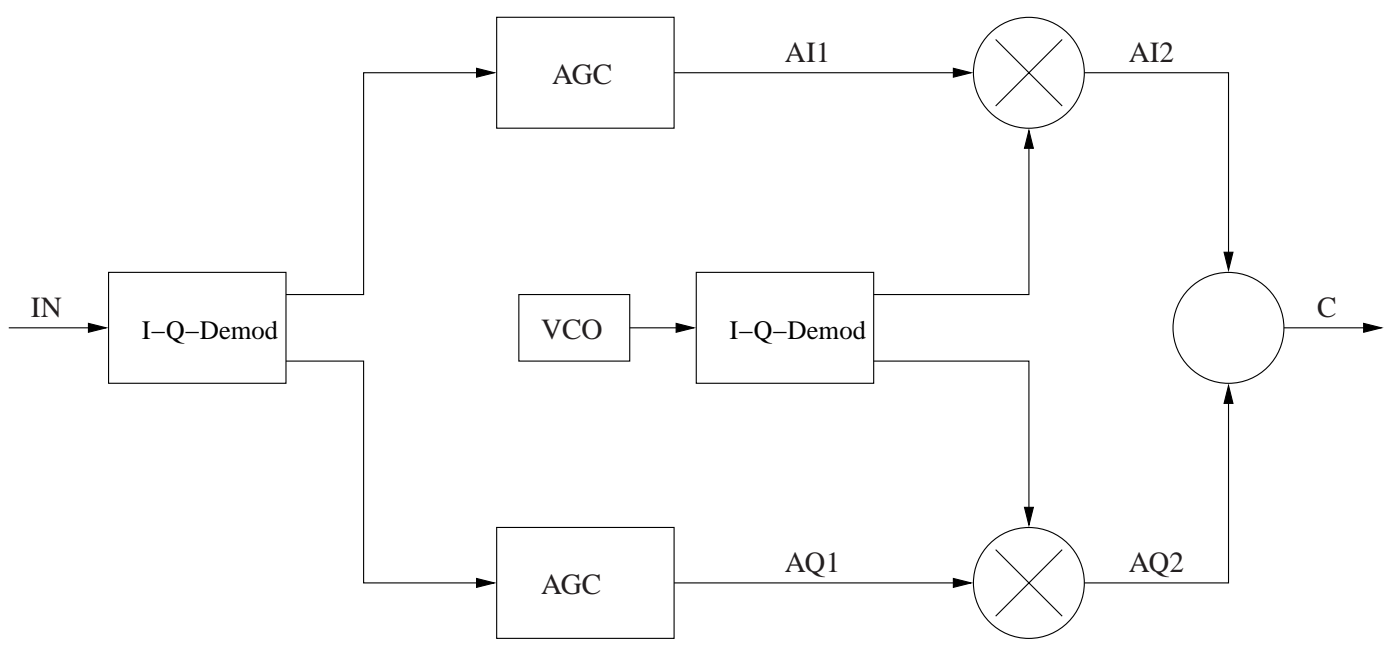

Fig. 1. Systemconcept: single sideband mixer.

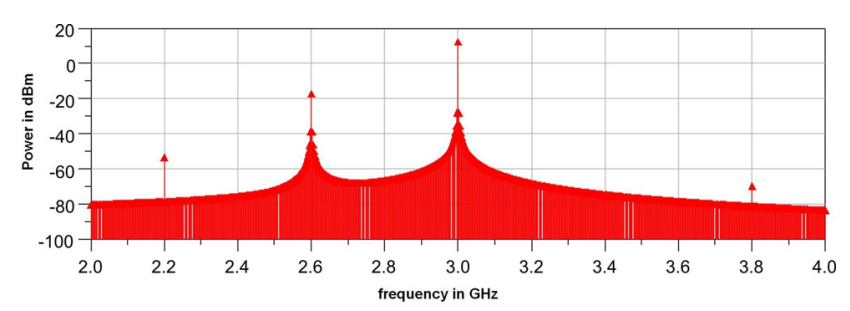

Fig. 2. Output spectrum of FFT during settling time.

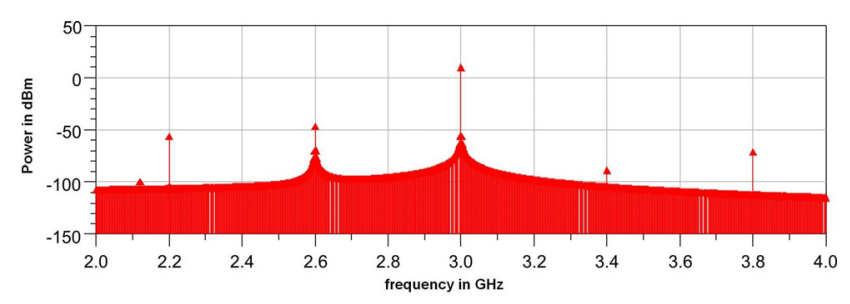

Fig. 3. Output spectrum of FFT after settling time.

and

$A_{Q 1}(t)=A_{Q 1} \cos \left(w_{\mathrm{in}} t+\Delta \Phi_{Q}\right)$.

After a few steps, the output signal

$C(t)=A_{I 1}(t)+A_{Q 1}(t)$

can be calculated for the upper and lower sideband

$\left|C_{\mathrm{USB}}\right|=\sqrt{a^{2}-b^{2}}$

$\left|C_{\mathrm{LSB}}\right|=\sqrt{a^{2}+b^{2}}$ where

$a=\frac{A_{Q 1} B_{Q} \sin \left(\Delta \Phi_{Q}\right)}{2}$

and

$b=\frac{A_{I 1} B_{I}}{2}+\frac{A_{Q 1} B_{Q} \cos \left(\Delta \Phi_{Q}\right)}{2}$.

In Fig. 5 the sideband suppression is shown in dependence on the relative phase error and the amplitude deviation in both channels.

Obviously both, an amplitude and a phase deviation have a strong influence on the sideband suppression. A phase error of $3^{\circ}$ approximately deteriotrates the signal as much as an amplitude error of about $2 \mathrm{~dB}$.

\section{Generation of a broad band I/Q signal}

There are a variety of possibilities to generate a broadband I/Q signal. Under consideration of the requirements of a wideband input spectrum and a very fast settling time and regarding previous results of the analysis of phase- and amplitude errors, three concepts have been proven as applicable.

\subsection{RC-CR phase shifter}

The first possibility to generate a $90^{\circ}$ phase difference, is an RC-CR combination. Identical resistors and capacitors assumed, this construction always provides a relative phase difference of $90^{\circ}$. With the transfer functions of the lowand highpass (Eqs. 5, 6) it can be shown, that the relative phase difference between this two filters remains $90^{\circ}$ over the whole frequency range.

$H_{\mathrm{TP}}=\frac{1}{1+j \omega R C}$ 

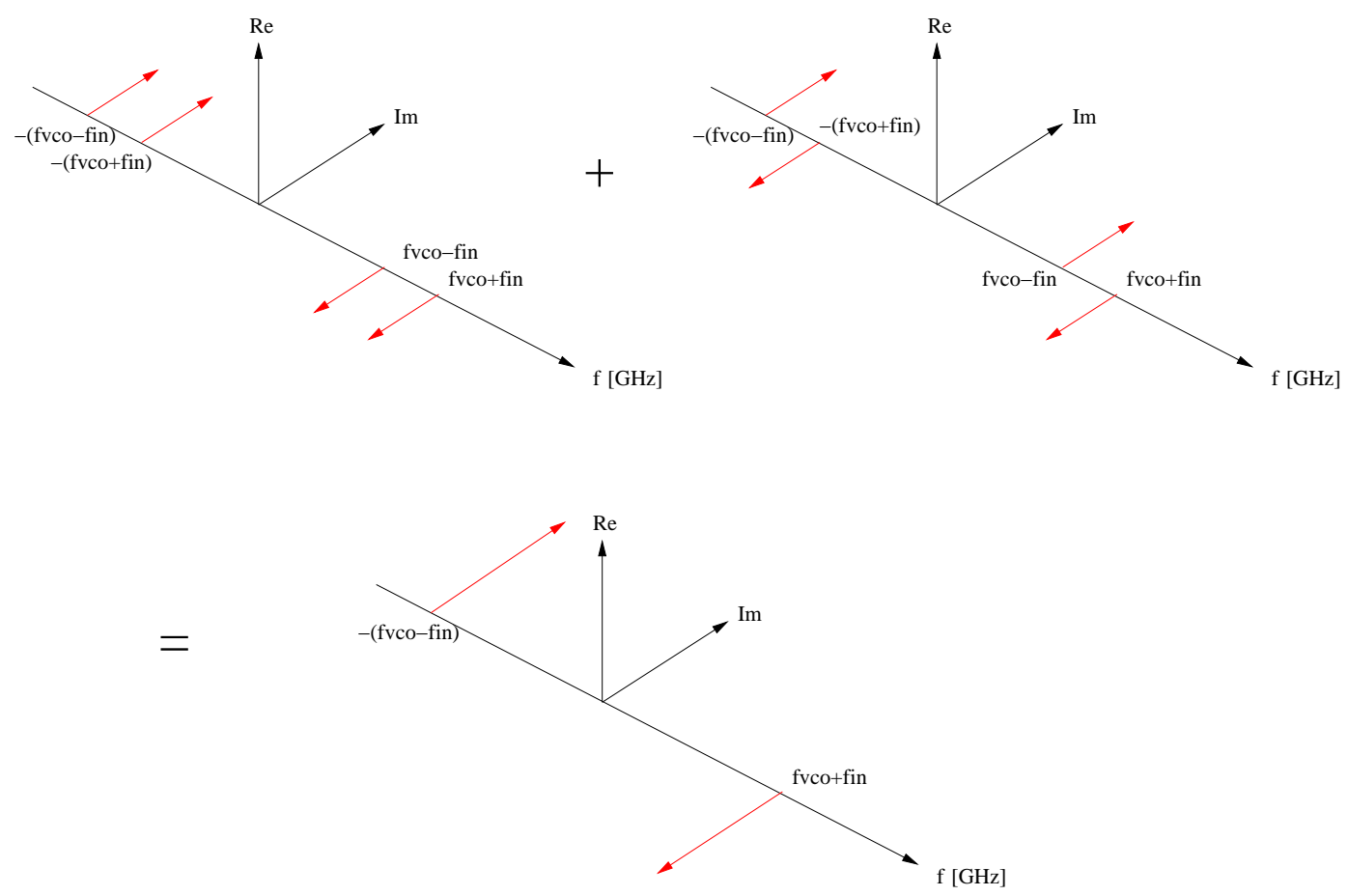

Fig. 4. Spectrum of the inphase and quadrature signals after frequency conversion.

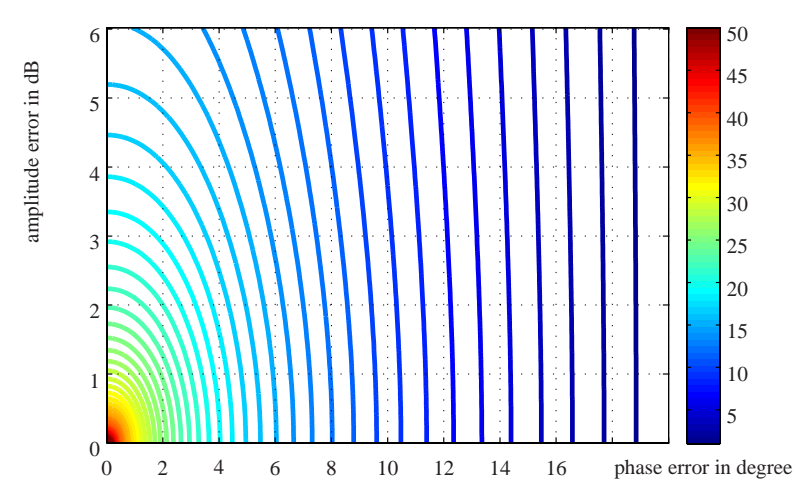

Fig. 5. Influence of phase- and amplitude errors on the side band suppression.

$H_{\mathrm{HP}}=\frac{j \omega R C}{1+j \omega R C}$

phase difference $=\angle H_{\mathrm{TP}}-\angle H_{\mathrm{HP}}=$

$-\arctan (w R C)-\frac{\pi}{2}+\arctan (w R C)=-90^{\circ}$

An issue of this type of phase shifter is the antipodal change of the amplitude levels in both signal channels. To achieve good results in sideband suppression the amplitudes of both signals have to be equal. This is done by an automatic-gaincontrol unit (AGC). In Fig. 6 the difference between the in-

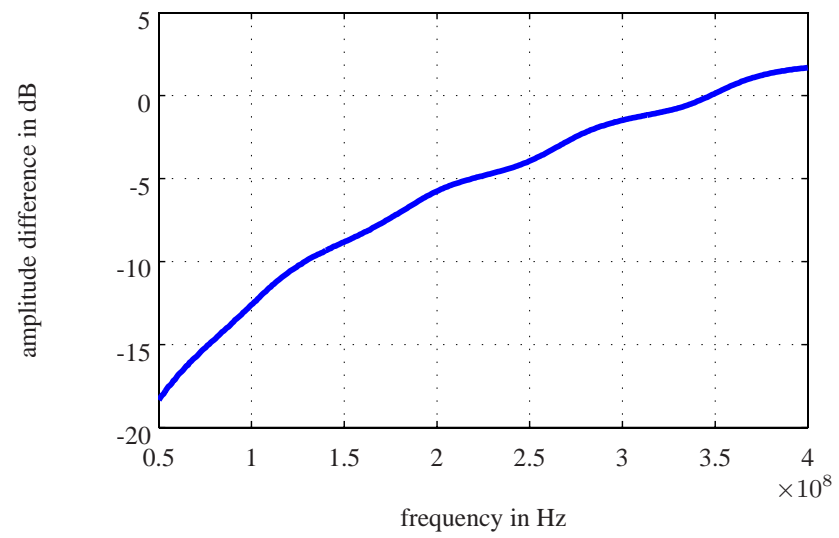

Fig. 6. Amplitude difference of I and Q channel of RC-CR element.

phase and quadrature channel is depicted. For a frequency step from $400 \mathrm{MHz}$ to $50 \mathrm{MHz}$ the maximum difference is approximately $18 \mathrm{~dB}$.

An increasing difference of the amplitudes causes a higher time constant of the AGC. To minimize the deterioration of the fast settling time, the amplitude influence of the phase shifter should be kept as small as possible. The phase response of this RC-CR component, which is designed for an input frequency range from $50 \mathrm{MHz}$ to $400 \mathrm{MHz}$, is shown in Fig. 7. 


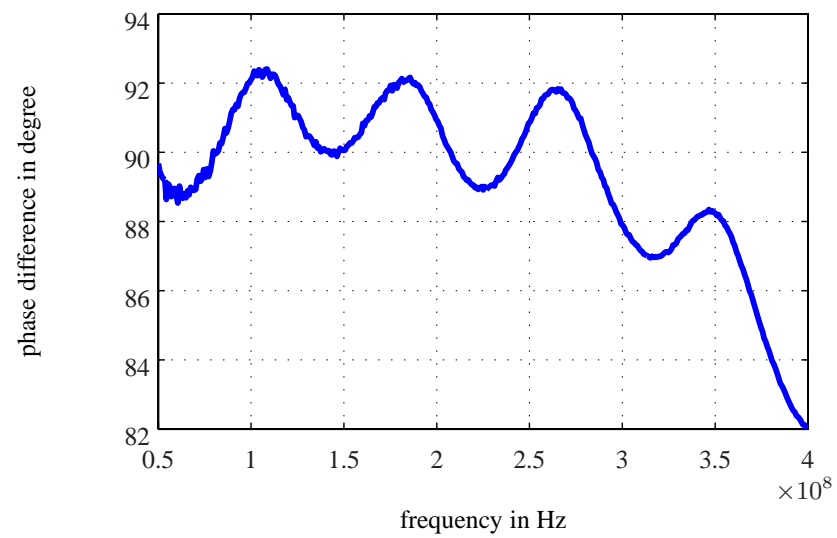

Fig. 7. Phase difference of I and Q channel of RC-CR element.

Up to a frequency of $360 \mathrm{MHz}$ the phase error remains smaller than $2.5^{\circ}$.

\subsection{Delay line phase shifter}

Another concept for phase shifting is shown in Fig. 8. The generation of a $90^{\circ}$ phase difference is realized exclusively with transducers and delay lines. Every transducer is used to split a single-ended signal into a differential signal. For mathematical description, the input signal is written vectorial.

$\mathbf{I N}(t)=e^{j \omega t}\left(\begin{array}{c}\cos \varphi \\ \sin \varphi\end{array}\right)=e^{j \omega t}\left(\begin{array}{l}1 \\ 0\end{array}\right)$

The input phase offset can be set to zero without further restrictions and the time dependence can be ignored. Assuming that the upper output of every balun is delayed by $180^{\circ} \mathrm{B} 1$ and $\mathrm{B} 2$ can be written as

$\mathbf{B 1}=\left(\begin{array}{c}\cos \left(\varphi_{B 1}=180^{\circ}\right) \\ \sin \left(\varphi_{\mathrm{B} 1}=180^{\circ}\right)\end{array}\right)=\left(\begin{array}{c}-1 \\ 0\end{array}\right)$

$\boldsymbol{B 2}=\left(\begin{array}{c}\cos \left(\varphi_{\mathrm{B} 2}=0^{\circ}\right) \\ \sin 0\left(\varphi_{\mathrm{B} 2}=0^{\circ}\right)\end{array}\right)=\left(\begin{array}{l}1 \\ 0\end{array}\right)$.

Each signal is divided by a transducer. The signals $\mathrm{C} 2$ and $\mathrm{C} 4$ are delayed and compared to $\mathrm{C} 1$ and $\mathrm{C} 3$. To get a relative phase difference of $90^{\circ}$ independent of frequency between the output signals Port 2 and Port3, the length of the delay line can be choosen arbitrarily. The amplitude difference of the output signals increases, if the electrical length of the delay line or phase difference between $\mathrm{C} 2$ and $\mathrm{C} 1$, and as well between $\mathrm{C} 4$ and $\mathrm{C} 3$, becomes smaller. With $\Delta \Phi_{\mathrm{C} 2}$ and $\Delta \Phi_{\mathrm{C} 4}$ being the phase differences caused by the delay lines $\mathrm{C} 2$ and $\mathrm{C} 4$, the output signals at Port 2 and Port 3 can be written as:

$$
\begin{aligned}
& \mid \text { Port2 }|=|\left(\begin{array}{c}
\cos \Delta \varphi_{\mathrm{C} 2}-1 \\
\sin \Delta \varphi_{\mathrm{C} 2}
\end{array}\right) \mid \\
& \mid \text { Port3 }|=|\left(\begin{array}{c}
\cos \Delta \varphi_{\mathrm{C} 4}+1 \\
\sin \Delta \varphi_{\mathrm{C} 4}
\end{array}\right) \mid
\end{aligned}
$$

Using the dot product (13), it can be shown that the signal vectors at the output ports are perpendicular to each other and therefore the phase difference is $90^{\circ}$, assumed that both delay lines cause the same phase difference.

Port2 • Port3 $=\cos ^{2}(\Delta \varphi)-1+\sin ^{2}(\Delta \varphi) \equiv 0$

The attenuation of the delay lines can be neglected and the phase difference of all balun-output-signals accounts for $180^{\circ}$. Due to the frequency dependence of the electrical length of the delay line (Pozar, 1998) the phase difference is

$\Delta \varphi=\frac{360^{\circ} \cdot l_{\text {delay }}}{\lambda}=\frac{360^{\circ} \cdot l_{\text {delay }} \sqrt{\epsilon_{r}} f}{c}$

Figure 9 shows the variation of the absolute amplitudes at Port2 and Port3, as well as the amplitude difference between the two output signals versus the phase delay caused by the delay lines.

To minimize the amplitude deviation of the output signals at a high frequency step it is necessary to scale the delay line. The phase delay should remain within $50^{\circ}$ to $150^{\circ}$ inside a frequency range of $50 \mathrm{MHz}$ to $400 \mathrm{MHz}$. Considering the attenuation of the delay lines Fig.10 visualizes the result.

Measurements of the transfer coefficients S21 are shown in Figs. 11 and 12. Figure 11 shows the absolute value of S21. A frequency step of $350 \mathrm{MHz}$ causes an amplitude deviation of $25 \mathrm{~dB}$.

Regarding Fig. 12 the measured phase deviation is up to $15^{\circ}$. Compared to theory and to the RC-CR module, this component shows a poorer behavior, since a lot of small degradations are accumulated.

\subsection{Active allpass as phase shifter}

The third possibilty generating an I/Q signal is an active allpass filter. The absolute value of the transfer function is a constant one what in turn makes an AGC dispensable. To maintain equivalent amplitude levels in both signal paths an active allpass filter is used to get a phase difference of $90^{\circ}$ at the output. But an allpass with a fixed capacitor generates a phase delay of $90^{\circ}$ at merely one frequency point. So the capacitor values of the allpass have to be controlled. In our circuit this is done by a varicap. In addition the allpass in the upper signal path only adds a phase offset to the signal, while the relative phase difference between the two signal paths is generated through the allpass in the lower signal path. The phase response of an allpass is

$$
\angle \mathrm{H}(j w)=-2 \arctan (w \mathrm{R} C)
$$

The required capacitor value of the varicap $C_{\mathrm{var}}$ can be calculated in order to get a phase delay of $90^{\circ}$ independent of the frequency.

$C_{\mathrm{var}}=\frac{-\left(\frac{\tan \left(\frac{\pi}{4}\right)}{2 \pi f R}\right) C_{\mathrm{fix}}}{\left(\frac{\tan \left(\frac{\pi}{4}\right)}{2 \pi f R}\right)-C_{\mathrm{fix}}}$ 


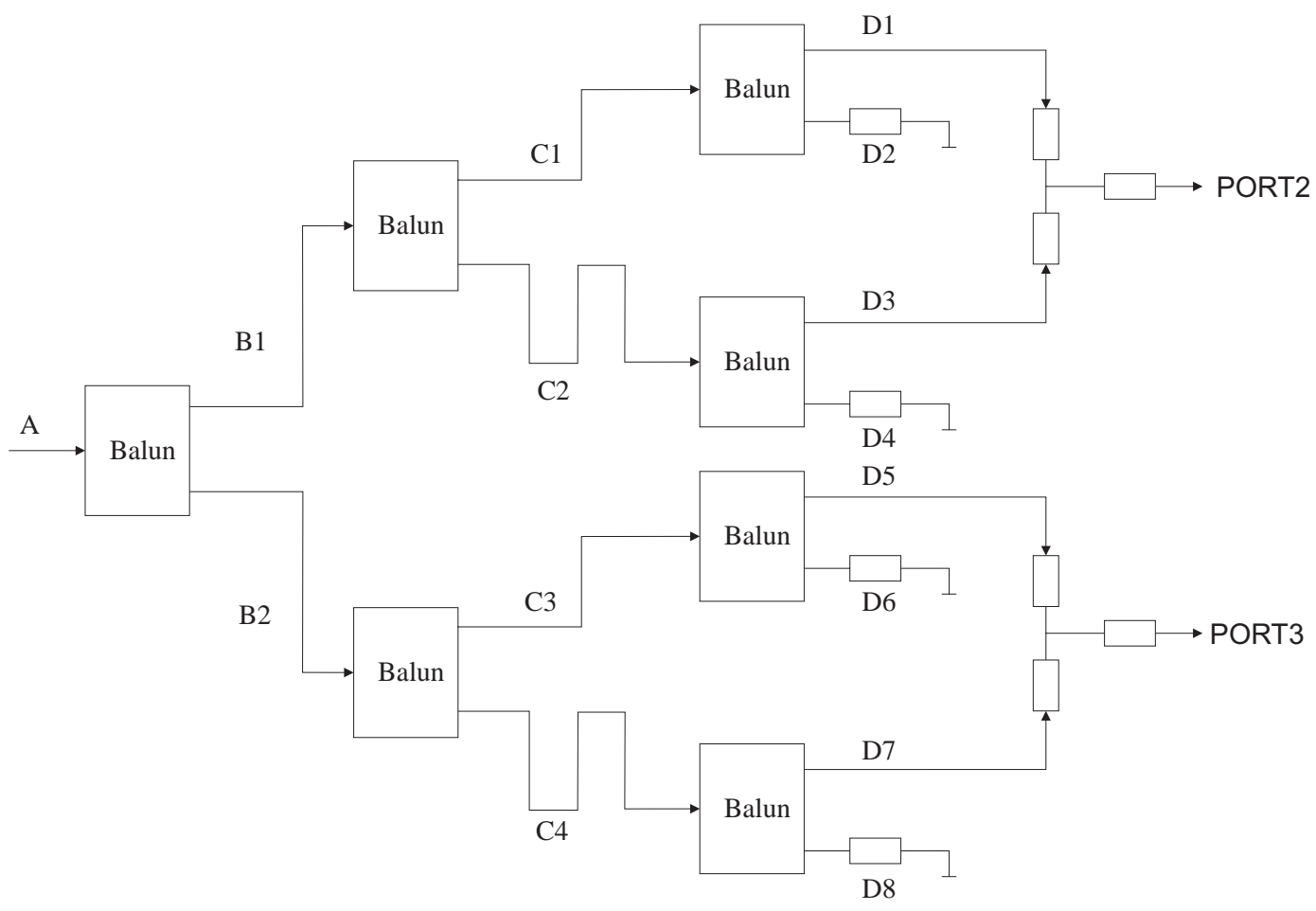

Fig. 8. Phase shifter consisting of transducers and delay lines.

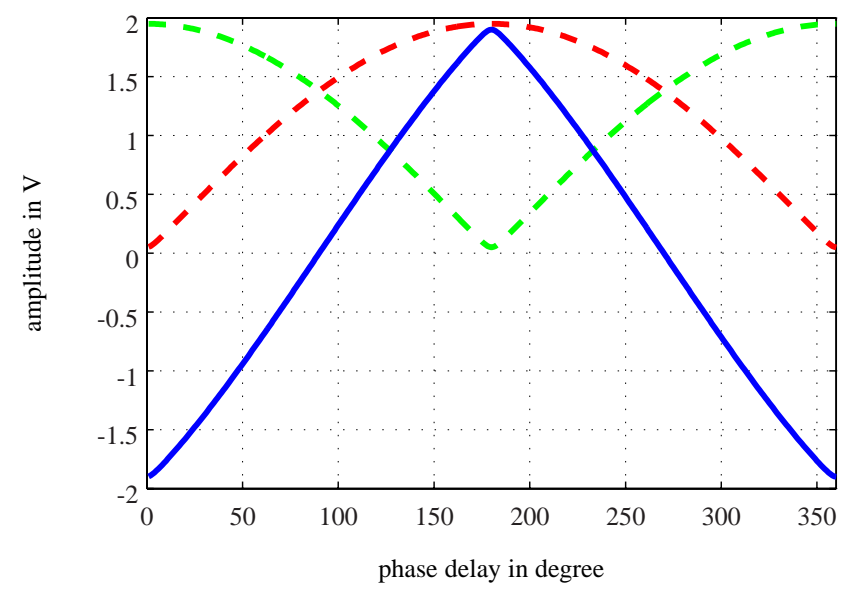

Fig. 9. Simulation of amplitude behaviour at the output of the phase shifter with delay lines, green dashed: PORT2, red dashed: PORT3, blue cont: difference.

To avoid a phase drift a closed loop control is used. The following nonlinear closed loop Eq. (17) adjusts the varicap to get a constant phase shift of $90^{\circ}$.

$\Delta \varphi\left(C\left(\mathrm{U}_{\mathrm{var}}\right)\right)=\Delta \varphi\left(C\left(\mathrm{PI}\left(\mathrm{U}_{90^{\circ}}-U_{\Delta \varphi}\right)\right)\right)$

where $\mathrm{PI}\left(\mathrm{U}_{90^{\circ}}-U_{\Delta \phi}\right)$ and $\left(C\left(U_{\text {var }}\right)\right)$ are the transfer functions of the PI-controller and the active allpass, respectively. The nonlinearity is a reason not to design a stand-alone solu-

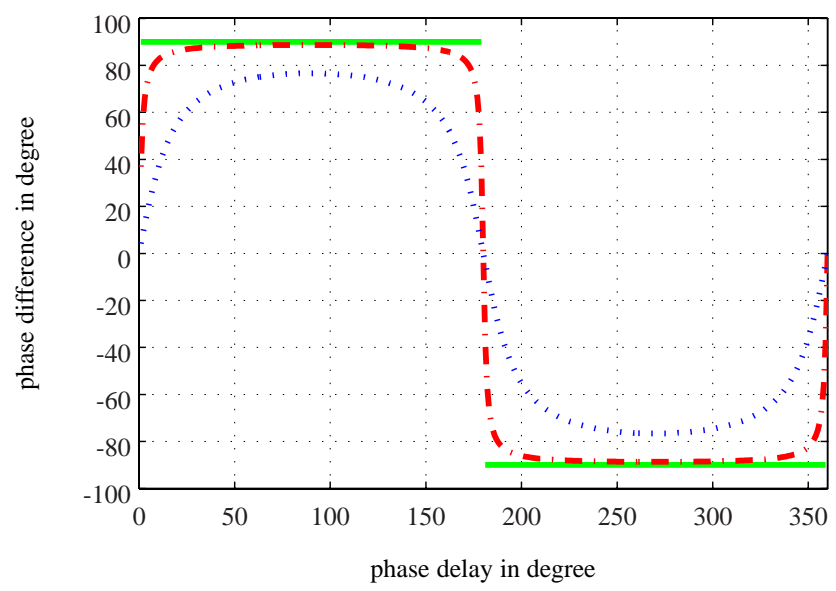

Fig. 10. Influence of attenuation of delay lines on phase difference between output signals, solid line: $0 \mathrm{~dB}$, dashed dotted: $0.1 \mathrm{~dB}$, dotted: $1 \mathrm{~dB}$.

tion with an allpass. But the transfer function of the allpass could be linearized if the control range of the varicap was smaller. This can be realized by a combination of an RC-CR element and an allpass. In this case the allpass only has to compensate a residual phase shift of about $5^{\circ}$.

Figure 13 shows the large signal schematic of the control loop. The control variable and the output variable is the same one $\varphi_{\text {out } 1}$. 


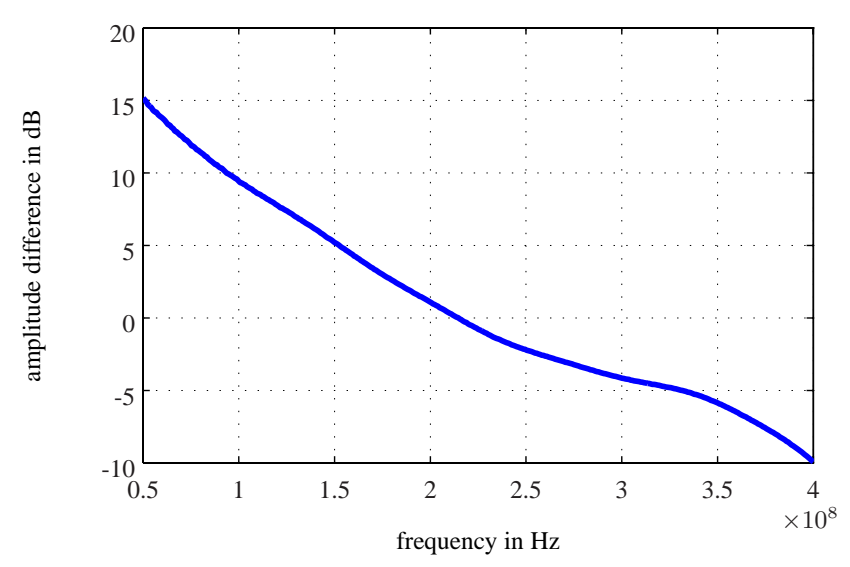

Fig. 11. Measured amplitude difference between the two output signals of the phase shifter with delay lines.

With an offset voltage $\mathrm{U}_{\text {off }}$ the operation point is adjusted. The phase delay can be written as

$\varphi_{\text {out } 1}\left(\mathrm{U}_{\text {var }}, f\right)=\varphi_{\text {out } 1}\left(\mathrm{U}_{\text {off }}+\mathrm{PI}\left(\mathrm{U}_{90^{\circ}}-\mathrm{U}_{\Delta \varphi}, \mathrm{f}\right)\right)$.

The transfer functions of the allpass and the phase detector have to be linearized. Linearizing the allpass the input signal $U_{\text {var }}$ can be written as

$\mathrm{U}_{\mathrm{var}}=\mathrm{U}_{\mathrm{var}_{0}}+\Delta \mathrm{U}_{\mathrm{var}}$.

The allpass transfer function differentiated with respect to $\Delta U_{\text {var }}$ yields

$\varphi_{\text {out } 1}\left(\mathrm{U}_{\mathrm{var}}, \mathrm{f}\right)=\varphi_{\text {out } 1}\left(\mathrm{U}_{\mathrm{var}}, \mathrm{f}\right)+\varphi_{\text {out } 1}^{\prime}\left(\mathrm{U}_{\mathrm{var}}, \mathrm{f}\right) \cdot \Delta \mathrm{U}_{\text {var }}$

in the operating point. The phase difference detected by the phase detector is

$$
\begin{array}{r}
\Delta \varphi\left(\mathrm{U}_{\mathrm{var}}, f\right)=\varphi_{\text {out } 1}\left(\mathrm{U}_{\mathrm{var}}, f\right)-\varphi_{\text {out } 2}\left(\mathrm{U}_{\mathrm{var}}, f\right)= \\
\varphi_{\text {out } 1}\left(\mathrm{U}_{\text {offset }}, f\right)+\Delta \varphi_{\text {out } 1}\left(\Delta \mathrm{U}_{\mathrm{var}}, f\right) \\
-\varphi_{\text {out } 2}\left(\mathrm{U}_{\text {offset }}, f\right)=\varphi_{\text {out } 1}^{\prime}\left(\mathrm{U}_{\mathrm{var}}, f\right) \cdot \Delta \mathrm{U}_{\mathrm{var}}
\end{array}
$$

Now the input signal of the PI-controller is

$\mathrm{U}_{\text {err }}(f)=\mathrm{U}_{90}-\mathrm{U}_{\Delta \varphi_{\text {off }}}-\mathrm{m} \cdot \Delta \varphi(f)$,

where $\mathrm{m} \cdot \Delta \varphi(f)$ ist the characteristic line of the phase detector. Taking into account the PI-controller the control voltage $\mathrm{U}_{\mathrm{var}}$ yields

$\mathrm{U}_{\mathrm{var}}(f)=\left(\mathrm{K}_{\mathrm{P}}+\frac{\mathrm{K}_{\mathrm{I}}}{\mathrm{s}}\right) \cdot \mathrm{U}_{\mathrm{err}}(f)+\mathrm{U}_{\text {off }}$

where $\mathrm{K}_{\mathrm{I}}$ and $\mathrm{K}_{\mathrm{P}}$ are the intergrated and the proportional part of the controller, respectively. Finally the transfer function of the control loop.

$H(s)=\frac{\Delta \varphi(f)}{\mathrm{W}(\mathrm{s})}=\frac{-\left(K_{\mathrm{P}}+\frac{K_{\mathrm{I}}}{s}\right) \varphi_{\text {out }}^{\prime}\left(\mathrm{U}_{\text {off }}, f\right) \mathrm{m}}{1+\left(K_{\mathrm{P}}+\frac{K_{\mathrm{I}}}{s}\right) \varphi_{\text {out }}^{\prime}\left(\mathrm{U}_{\text {off }}, f\right) \mathrm{m}}$

where $W(s)$ is an interference signal concerning the phase difference. It is used to simulate the change of phase difference between the two channels.

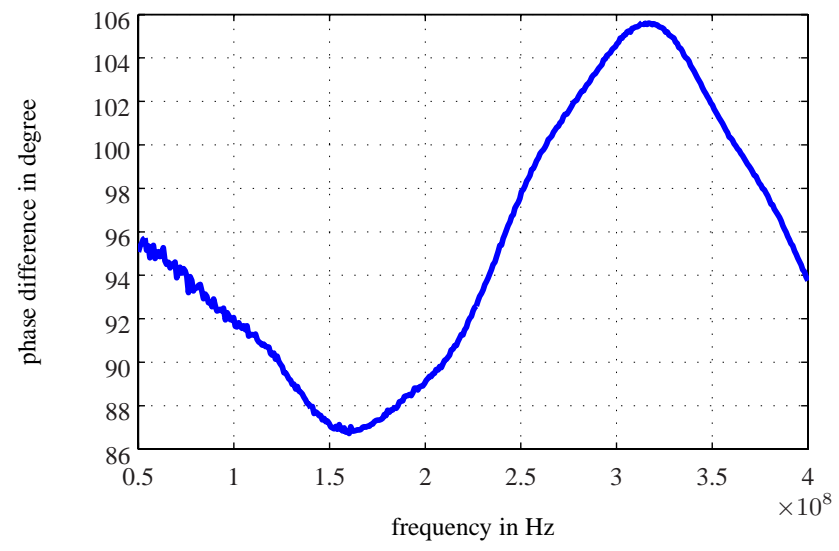

Fig. 12. Measured phase difference between the two output signals of the phase shifter with delay lines.

\section{Frequency conversion and signal superposition}

For upconverting the frequency in both signal paths a mixer (Fig. 1) with a wide output spectrum and a high LO suppression was chosen. The LO consists of a VCO with a PLL. The phase difference of $90^{\circ}$ is done by a discrete power splitter and the superposition of the signals at the output by a resistive combiner.

\section{Simulation and measurements}

\subsection{Phase stability}

The control loop was simulated in Matlab (Angermann and Beuschel, 2005). A settling time lower than $3 \mu$ s can be achieved. Figure 14 shows the control behaviour after an applied phase deviation, that is similar to a frequency step of $350 \mathrm{MHz}$ at the input. The time delay to adjust the manipulated phase to $90^{\circ}$ can be seen clearly.

\subsection{Measurement of side band suppression}

The setup for the measurement is as follows: The input signal is splitted by a resistive divider. For the inphase and quadrature signal generation an RC-CR filter is used followed by an AGC with a settling time below $3 \mu \mathrm{s}$. An allpass as described in Sect. 4.3 is resigned but could improve the results. The achievable sideband suppression is shown in Fig. 15.

\section{Conclusions}

We designed a broadband single side band mixer with a very short settling time. The modular setup allows simple changing. For generating an I/Q signal with high bandwidth three methods have been analyzed. An RC-CR element, a phase shifter with transducers and delay lines and finally an active allpass filter. Each individual method has its advantages but 


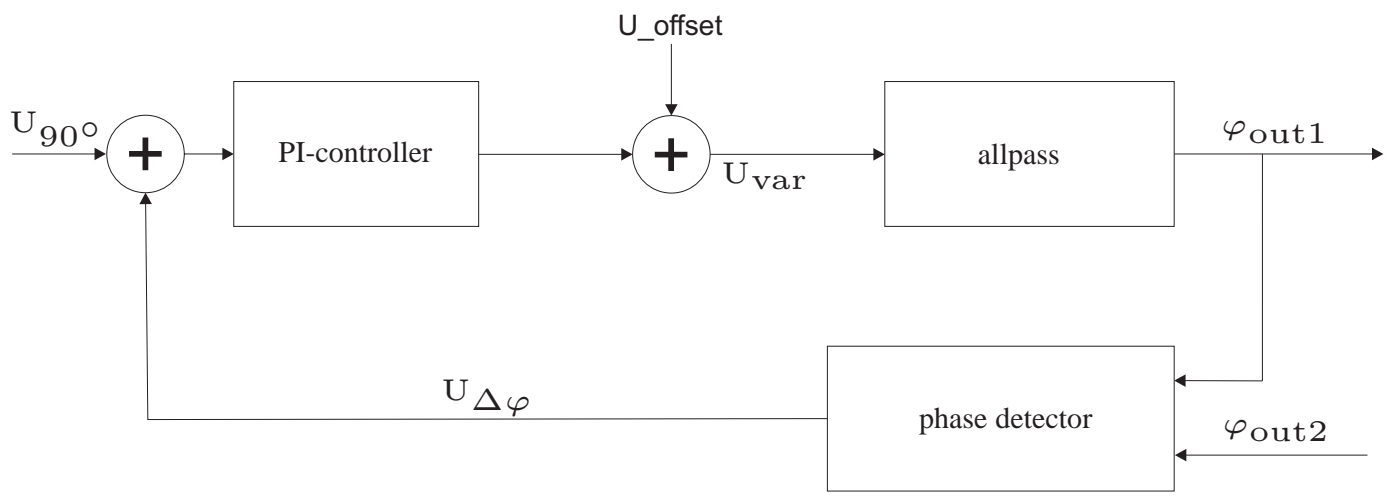

Fig. 13. Control loop of the active phase shifter.

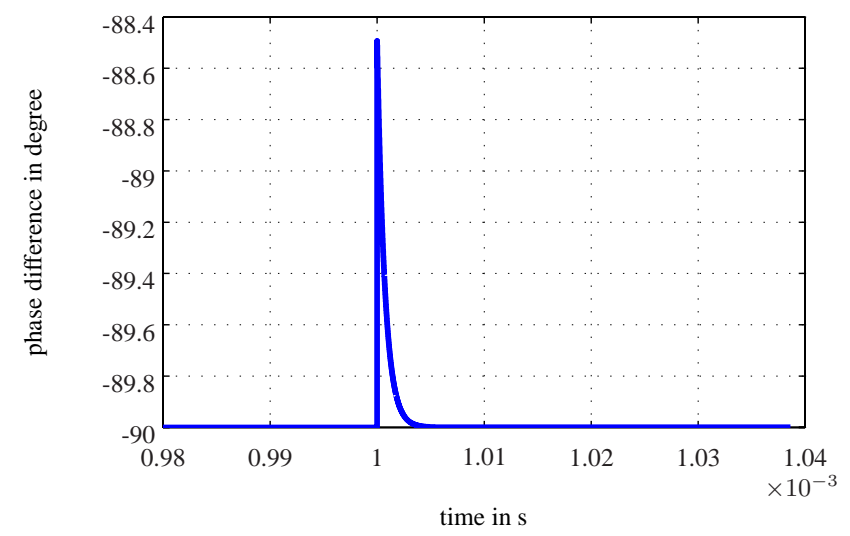

Fig. 14. Matlab/Simulink simulation of the small signal control loop.

the RC-CR filter was chosen, since the phase error is best inside the required bandwidth and equalizing the amplitudes up to a difference of $18 \mathrm{~dB}$ can be obtained by a fast AGC. In combination with an active allpass filter the phase error could be even improved. The described combination was build up and evaluated. It is shown, that a settling time of less than $3 \mu \mathrm{s}$ is possible within the bandwidth of $400 \mathrm{MHz}$. Hence the signal source can be used as synthsizer for stepped frequency radar and could even be used as a hopped frequency signal source.

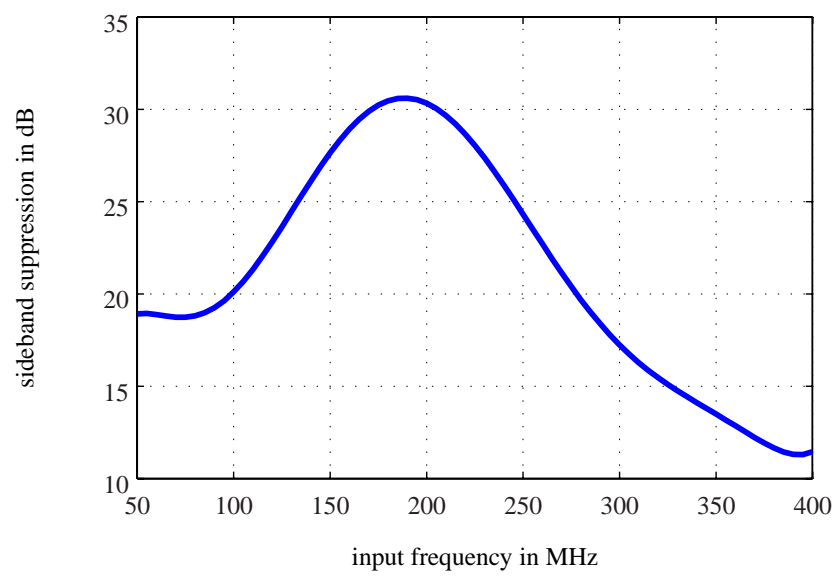

Fig. 15. Sideband suppression with $\mathrm{VCO}=2.8 \mathrm{GHz}$.

\section{References}

Angermann, A. and Beuschel, M. R. U. W.: Matlab - SimulinkStateflow, 4th edn., 2005.

Haßler, S.: Entwicklung eines breitbandigen Einseitenbandmischers mit sehr kurzer Einschwingzeit, 2009.

Pozar, D. M.: Microwave Engineering, 2nd edn., 1998. 